
\title{
CSResearch Square \\ Generation of Dystrophin Short Product-Specific Tag-Insertion Mouse: Distinct Dp71 Glycoprotein Complexes at Inhibitory Postsynapse and Glia Limitans
}

\author{
Takahiro Fujimoto ( $\nabla$ tfuji@koto.kpu-m.ac.jp) \\ Kyoto Prefectural University of Medicine: Kyoto Furitsu Ika Daigaku https://orcid.org/0000-0002-9975-7008 \\ Takeshi Yaoi \\ Kyoto Prefectural University of Medicine: Kyoto Furitsu Ika Daigaku \\ Kenta Nakano \\ National Center for Global Health and Medicine: Kokuritsu Kenkyu Kaihatsu Hojin Kokuritsu Kokusai Iryo Kenkyu Center \\ Tetsuya Arai \\ National Center for Global Health and Medicine: Kokuritsu Kenkyu Kaihatsu Hojin Kokuritsu Kokusai Iryo Kenkyu Center \\ Tadashi Okamura \\ National Center for Global Health and Medicine: Kokuritsu Kenkyu Kaihatsu Hojin Kokuritsu Kokusai Iryo Kenkyu Center \\ Kyoko Itoh \\ Kyoto Prefectural University of Medicine: Kyoto Furitsu Ika Daigaku https://orcid.org/0000-0001-8369-8800
}

\section{Research Article}

Keywords: Dp71, dystrophin, transgenic mouse, inhibitory postsynapse, glia limitans, immunohistochemical staining

Posted Date: December 10th, 2021

DOI: https://doi.org/10.21203/rs.3.rs-1091068/v1

License: (1) This work is licensed under a Creative Commons Attribution 4.0 International License. Read Full License

Version of Record: A version of this preprint was published at Cellular and Molecular Life Sciences on January 30th, 2022. See the published version at https://doi.org/10.1007/s00018-022-04151-2. 


\section{Abstract}

Duchenne muscular dystrophy (DMD), the most severe form of dystrophinopathies, is a fatal X-linked recessive neuromuscular disorder characterized by progressive muscle degeneration and various extents of intellectual disabilities. Physiological and pathological roles of the responsible gene, dystrophin, in the brain remain elusive due to the presence of multiple dystrophin products, mainly full-length dystrophin, Dp427, and the short product, Dp71. In this study, we generated a Dp71-specific hemagglutinin (HA) peptide tag-insertion mice to enable specific detection of intrinsic Dp71 expression by anti-HA tag antibodies. Immunohistochemical detections in the transgenic mice demonstrated Dp71 expression not only at the blood-brain barrier, where astrocytic endfeet surround the microvessels, but also at the inhibitory postsynapse of hippocampal dentate granule neurons. Interestingly, hippocampal cornu ammonis (CA) 1 pyramidal neurons were negative for Dp71 although Dp427 detected by anti-dystrophin antibody was clearly present at the inhibitory postsynapse, suggesting cell-type dependent dystrophin expressions. Precise examination using the primary hippocampal culture validated exclusive localization of Dp71 at the inhibitory postsynaptic compartment but not at the excitatory synapse in neurons. We further performed interactome analysis and found that Dp71 formed distinct molecular complexes, i.e. synapse-associated Dp71 interacted with dystroglycan (Dg) and dystrobrevinb (Dtnb) whereas glia-associated Dp71 did with Dg and dystrobrevina (Dtna). Thus, our data indicates that Dp71 and its binding partners are relevant to the inhibitory postsynaptic function of hippocampal granule neurons and the novel Dp71-transgenic mouse provides a valuable tool to understand precise physiological expressions and functions of Dp71 and its interaction proteins in vivo and in vitro.

\section{Introduction}

Dystrophin is the causative gene for X-linked recessive Duchenne/Becker muscular dystrophy (DMD/BMD) and consists of 79 exons over 2.2Mb of genomic DNA [1-5]. Although fatal clinical symptom of DMD is progressive muscle degeneration, non-muscular manifestations have also been described, including cognitive impairments, epilepsy and retinal electrophysiology [6-12]. Due to several intragenic gene promoters, dystrophin produces multiple forms of dystrophin in tissue-type dependent manner $[6,9,12]$. In muscles, the full-length dystrophin (known as Dp427) functions as a dystrophin glycoprotein complex by interacting with dystroglycan, dystrobrevins, syntrophins, and sarcoglycans, which is essential for the structural integrity and physiological function of myocytes $[6,9,13]$. In brains, Dp427 and the C-terminal short product, Dp71, which is transcribed from intron 62 and encoded by exons 63 to 79, are expressed before and after birth [14-22] and its defect is suspected to associate with cognitive impairments in DMD [6, 9]. Indeed, Dp71-deficient mice demonstrated neurological symptoms [23], while physiological expression and function of Dp71 remain elusive. Growing evidence indicated Dp427 expression at inhibitory postsynapses both in the cerebellum Purkinje cells and in the hippocampal pyramidal cells [18, 20, 22, 24], and Dp71 expression at glial foot processes surrounding blood-brain barrier (BBB) [24-27], however, further investigations for dystrophin expression and function in brain are necessary to unveil the molecular basis of DMD. A major difficulty in dystrophin studies is that it is almost impossible to detect exclusively Dp71 by immunohistological techniques because the primary amino acid sequence of Dp71 except its hexapeptide at the $\mathrm{NH}_{2}$-terminus is identical with them of longer dystrophin products. Additionally, multiple Dp71 splicing isoforms, such as Dp71d, Dp71f and Dp40 shortest isoform, are potentially expressed from the intron 62 promoter $[9,28,29]$.

In order to overcome this difficulty, we generated herein a transgenic mouse line that enable specific detection of intrinsic Dp71 protein by genetically inserting DNA fragment encoding HA-tag peptide at the N-terminus of Dp71 in-frame. Successfully, endogenous Dp71 protein was detected in the transgenic mouse brains by anti-HA tag antibodies in both biochemical and histological techniques. Distinct Dp71 isoforms, Dp71d and Dp71f, were evidently present in the adult brains but Dp40 was not. Dp71 was prominently detected at glia limitans at microvessels as well as pia membranes in accordance with the previous observations. It is noteworthy that Dp71 was detected at inhibitory postsynapse compartment within the perisomatic and dendritic areas of hippocampal dentate granule neurons in vivo. Further assessment using primary hippocampal neuronal culture validated exclusive localization of Dp71 at inhibitory postsynapse compartment. Importantly, this transgenic mouse line was applied to biochemical assay, immunoprecipitation followed by mass spectrometrical identification, which demonstrated dystrobrevins, syntrophins and Insyn1 as Dp71-interaction proteins in the adult hippocampi.

\section{Materials And Methods}

\section{Generation of Dp71-specific HA-tag insertion mice}

CRISPR/Cas9-mediated genome editing was performed as described previously [30]. Briefly, the crRNA guiding sequence for the intron 62 of dystrophin (Dmd) was designed as follows: 5'-GTGTTCCCTCATGGTTGTAA-3'. Both crRNA and tracrRNA were synthesized 
artificially (Fasmac, Kanagawa, Japan). For generating HA-tag insertion mice, the recombinant Cas9 protein (50 ng/mL, New England Biolabs, MA, USA), crRNA (1.22 mol $\mu \mathrm{L})$, tracrRNA $(1.22 \mathrm{~mol} / \mu \mathrm{L})$, and single strand oligonucleotide $\left(100 \mathrm{ng} / \mu \mathrm{L}: 5^{\prime}\right.$ -

GTCCGCCCCTCACTGCCTGTGAAACCCTTACAACCATGTACCCATACGATGTTCCAGATTACGCTATGAGGGAACACCTCAAAGGGTAAGTGGAT-

$\left.3^{\prime}\right)$ were coinjected into the cytoplasm of pronuclear stage eggs from B6D2F1 mice. Two-cell stage embryos were transferred into the oviduct of pseudopregnant ICR mice anesthetized using the mixture of $0.75 \mathrm{mg} / \mathrm{kg}$ medetomidine, $4.0 \mathrm{mg} / \mathrm{kg}$ midazolam, and 5.0 $\mathrm{mg} / \mathrm{kg}$ butorphanol during operation [31]. The precise genetic modification was validated by genomic DNA sequencing. All mice were bred and maintained under specific pathogen-free conditions in our animal facility.

\section{Genotyping PCR and Genomic DNA sequencing}

Genotyping for HA-tagged Dp71 allele was performed using DNA isolated from tails using the following protocols: Forward primer, 5'agccccgtggcttgggcaagcttac-3'; Reverse primer, 5'-cttaccctttgaggtgttccctcat-3'; Reaction conditions, $95^{\circ} \mathrm{C}$ for 2 min followed by 34 cycles of $98^{\circ} \mathrm{C}$ for $10 \mathrm{~s}, 60^{\circ} \mathrm{C}$ for $30 \mathrm{~s}, 68^{\circ} \mathrm{C}$ for $8 \mathrm{~s}$, with a final step at $72^{\circ} \mathrm{C}$ for $5 \mathrm{~min}$. Expected band sizes 100 base pairs for wild-type and 130 base pairs for transgenic mice. Purified PCR products were subjected to DNA sequencing.

\section{Antibodies}

The antibodies used were described in Suppl. Table S1.

\section{Immunoprecipitations and Western blotting}

Immunoprecipitation and western blotting were performed as described previously with slight modifications [32]. Briefly, total protein lysates were obtained by extracting mouse tissues in the lysis buffer A ( $25 \mathrm{mM}$ Tris- $\mathrm{HCl}, \mathrm{pH} 7.5,150 \mathrm{mM} \mathrm{NaCl}, 1 \%$ Nonidet P-40, 0.5\% sodium deoxycholate, 0.1\% SDS, 5 mM EDTA, and Protease Inhibitor Cocktail for Use with Mammalian Cell and Tissue Extracts). For simple-immunoprecipitations and co-immunoprecipitations, tissues or cells were extracted in the lysis buffer $A$ and the lysis buffer $B$ (25 mM Tris-HCl, pH7.5, 150 mM NaCl, 1\% Nonidet P-40, 5 mM EDTA, and Protease Inhibitor Cocktail for Use with Mammalian Cell and Tissue Extracts), respectively, and then the lysates were incubated with Pierce ${ }^{\mathrm{TM}}$ Anti-HA Magnetic Beads (Thermo Fisher Scientific). After 4 times washing with the appropriate lysis buffer, immunoprecipitates were subjected to SDS-PAGE and western blotting.

\section{Ectopic expressions of Dp71d and Dp71f in HEK293T cells}

The N-terminal HA-tagged mouse Dp71d (residues 1-617) or the N-terminal HA-tagged mouse Dp71f (residues 1-622) expression vectors were transfected into HEK293T cells as described previously [32].

\section{Immunocytochemistry}

The homozygous TG mouse brains or WT mouse brains (8-12 weeks old) were fresh frozen in Tissue-Tek O.C.T. Compound and subjected to preparation of coronal sections. Immunocytochemical staining was performed as described previously with slight modification [32, 33]. Fluorescence images were acquired by a confocal fluorescence microscope (Zeiss LSM510 Ver. 4.0 or Zeiss LSM900 with Airyscan, Oberkochen, Germany). Z-stack of confocal microscope images are turned into a single 2D image by using a maximum intensity projection function of Zeiss ZEN2009 software. Multiple image tiles were taken with a 10 'objective to cover the targeted region in each section and the tiles were obtained apposing each other with 10\% overlapping by using Zeiss LSM 900 with Airyscan. The stitched images were created by the software ZEN 3.2 (Carl Zeiss, Oberkochen, Germany). Synapse numbers were measured with ImageJ 1.53e software and ZEN 3.2.

\section{Primary culture}

Cultured hippocampal neurons and cultured non-neuronal glial cells were prepared from homozygous HA-Dp71 transgenic mouse embryos (Embryonic day-18) or postnatal day-0 pups as described previously [33].

\section{Sample preparation for mass spectrometry}

The immunoprecipitates were reduced and alkylated in $10 \mathrm{mM}$ dithiothreitol (GE healthcare) and $50 \mathrm{mM}$ iodoacetamide (Sigma-Aldrich), respectively. Proteins were digested using trypsin (Sequencing Grade Modified Trypsin V5111, Promega) suspended in $50 \mathrm{mM}$ ammonium bicarbonate (FUJIFILM Wako). The digested samples were concentrated in a centrifugal concentrator CC-105 (TOMY) and resuspended in $0.1 \%(\mathrm{v} / \mathrm{v})$ formic acid (FUJIFILM Wako). 


\section{LC-MS/MS sample analysis}

LC-MS/MS analyses were performed by nano LC (UltiMate® 3000) (Dionex, Sunnyvale, CA, USA) coupled with Q Exactive plus orbitrap mass spectrometer (Thermo Scientific, Waltham, MA, USA). Instrument operation and data acquisition were performed using Xcalibur Software (Thermo Scientific, Waltham, MA, USA). Digested peptides were separated on a $500 \times 0.075 \mathrm{~mm}$ capillary reversed-phase column (CERI, Tokyo, Japan) at a flow rate of $400 \mathrm{~nL} / \mathrm{min}$ and a column temperature at $60^{\circ} \mathrm{C}$. The mobile phase was composed of water with $0.1 \%(\mathrm{v} / \mathrm{v})$ formic acid (eluent $\mathrm{A}$ ) and of $80 \%$ acetonitrile with $0.1 \%(\mathrm{v} / \mathrm{v})$ formic acid (eluent $\mathrm{B})$. The peptides were separated by a linear gradient of eluent B up to $35 \%$ in 210 minutes for a 4 hours gradient run. The Q Exactive was operated in data dependent (dd) mode with full scans acquired at a resolution of 35,000 at $350 \mathrm{~m} / \mathrm{z}$ and with dd-MS/MS scans acquired at a resolution of 17,500. The mass spectrometer was operated in positive mode in the scan range of $350 \sim 1,500 \mathrm{~m} / \mathrm{z}$. Fixed first $\mathrm{m} / \mathrm{z}$ is 150 in dd-MS/MS scans. Up to the top 10 most abundant isotope patterns with a charge $\geq 2$ from the survey scan were selected with an isolation window of $1.6 \mathrm{~m} / \mathrm{z}$. The maximum ion injection times for the full scan and the dd-MS/MS scans were $20 \mathrm{~ms}$ and $100 \mathrm{~ms}$ respectively, and the automatic gain control (AGC) for the full scan and the dd-MS/MS scans were 3E6 and 1E5 respectively. Repeat sequencing of peptides was kept to a minimum by dynamic exclusion of the sequenced peptides for $20 \mathrm{~s}$.

\section{LC-MS/MS data analysis}

The raw MS/MS data were processed using Mascot version 2.6.0 (Matrix Sciences), and the cysteine carbamidomethylation was used as a static modification, the oxidation of methionine as a dynamic modification, a precursor mass tolerance of $0.5 \mathrm{Da}$, and a fragment mass tolerance of 0.1 Da. The obtained .dat files of all fractions were processed by Scaffold version 5.0.1 (Proteome Software Inc.). The parameters of Scaffold were adjusted to assure the precise identification of peptides and proteins as below: Protein identifications were accepted if they could be established at greater than $99 \%$ probability and peptide identifications were accepted if greater than $90 \%$ (using Scaffold Local FDR algorithm) contained at least 1 identified peptide. The normalized spectral abundance factor (Weighted spectra) was calculated for each protein and the relative abundances were compared between transgenic mouse hippocampus sample and wild-type mouse hippocampus sample. Proteins with weighted spectra more than 10 were considered as significant proteins and the quantitative differences were analyzed by Fisher's exact test through Scaffold. Values of $p<0.05$ were considered statistically significant.

\section{Results}

\section{Generation of Dp71-specific tag-insertion mice}

We generated a Dp71-specific HA peptide tag-insertion mouse line to enable specific detection of intrinsic Dp71 expression by anti-HA tag antibodies. In this mouse line, the short stretch of 30 nucleotide bases encoding HA peptide tag was inserted at the Dp71-specific translation initiation site which is present in the intron 62 of dystrophin gene to fuse with the N-terminal amino acid sequence of Dp71 in-frame (Fig. 1a). Both female and male homozygous transgenic (TG) mice were generated by intercrossing male TG mice with female heterozygous TG mice (Suppl. Fig. 1) and used in this study. To validate specific detection of endogenous Dp71 protein expressions in the TG mouse tissues, we performed western blotting and immunoprecipitations using anti-HA tag antibody. Anti-HA tag antibody revealed a significant signal for HA-Dp71 expression at $71 \mathrm{kDa}$ in the cerebrum extract from TG mice but not in the WT control and muscle extracts (Fig. 1b), although anti-dystrophin antibody detected not only Dp71 but also Dp427 in both WT and TG tissues. HADp71 expressions were comparably detected among cerebrum, hippocampus, and cerebellum extracts from TG mice (Fig. 1c). Enrichment of HA-Dp71 protein by immunoprecipitation using anti-HA magnetic beads revealed low level of HA-Dp71 expression in the muscle extract derived from TG mice (Fig. 1d). As the gene promoter within the intron 62 of dystrophin gene potentially produce multiple short dystrophins, Dp71 and Dp40, as well as its splicing variants such as Dp71d and Dp71f $[9,28]$, we analyzed whether mouse brain would express Dp40, Dp71d and Dp71f. Dp71d and Dp71f proteins ectopically expressed in HEK293T cells were used as the positive controls, and then immunoprecipitates by anti-HA beads from the HEK293T cell extracts and mouse cerebrum tissue extracts were blotted by anti-HA, -Pan dystrophin, -Dp71d, or -Dp71f antibodies (Fig. 1e). Anti-HA beads provided specific immunocapture and detection of Dp71 including both Dp71d and Dp71f isoforms from TG-cerebrum, while the other shorter dystrophin products such as Dp40 were not detected. Thus, Dp71 is only dystrophin product derived from intron 62 gene promoter in the physiological mouse brains as far as we examined. Taken together, Dp71-specific tag-insertion mouse line was successfully established and the tag-detection potentially provides endogenous expression profile of Dp71. 


\section{Ha-tagged Dp71 Expression In The Adult Mouse Brain}

Double immunohistochemical detection of HA-Dp71 with Prox1, a hippocampal granule neuronal marker, in the adult TG mouse hippocampal regions demonstrated that Dp71 was highly expressed in the dentate gyrus compared to the cornu ammonis (CA) 1 area (Fig. 2a). The signal specificity for HA-Dp71 was validated by comparing with the immunoreactivity against wild-type (WT) control section in which no signal was observed (Suppl. Fig. 2a). HA-Dp71 was moderately detected in the TG mouse cerebral cortex whereas there was little staining of the corpus callosum. Dp71 is well-known as a dystrophin short product present at the astrocytic endfeet surrounding BBB and attaching to the pia mater. Consistently, HA-Dp71 was detected along BBB and pia mater labelled by GFAP throughout the brain including hippocampus and cerebral cortex (Fig. 2b). HA-Dp71 signals were restricted to the glia limitans within the GFAP-positive astrocytes. Since dystrophin functions as a molecular complex with dystroglycan (Dg), we checked colocalization of HADp71 with bDg in the TG hippocampus and cerebral cortex (Fig. 2c and Suppl. Fig. 2b). HA-Dp71 signals were well-colocalized with bDG at BBB and pia mater in the TG sections, whereas no HA-signal was observed in the WT sections. Similarly, syntrophin (Snt) and dystrobrevina (Dtna), well-known components of dystrophin-Dg macromolecular complex, were detected at the HA-Dp71-positive BBB structures in the TG cerebral cortex and hippocampus (Suppl. Fig. 3). Thus, immunohistochemical detections of HA-Dp71 in the TG brain sections clearly showed Dp71 expressions not only at glia limitans but also at hippocampal dentate gyrus.

\section{HA-Dp71 expression in the inhibitory postsynapse of the hippocampal dentate gyrus}

To address the HA-Dp71 expression in the dentate gyrus, we performed double-immunohistochemical detections of HA-Dp71 with gephyrin, bDg, and PSD95. HA-Dp71 was detected as puncta and partially co-labeled with gephyrin, an inhibitory postsynapse marker, in the hippocampal dentate gyrus granular layer (Fig. 3a) and the molecular layer (Fig. 3b). In the dentate gyrus, HA-Dp71 signals were also co-labeled with bDg (Fig. 3c) but not with PSD95, an excitatory postsynapse marker (Fig. 3d). Although HA-Dp71 were present at both synapses and glia limitans in the dentate gyrus, Dys1 antibody which recognizes rod domain specific for Dp427 revealed no specific signal in the dentate gyrus region (Fig. 3e). As goat anti-mouse secondary antibody showed weak non-specific fluorescence within blood vessels and intracellular granules on mouse tissues (Fig. 3f), we ignored such signals as background noises. Taken together, dystrophin product expressed in the dentate gyrus was Dp71, and Dp71-Dg complex appeared to be located at the inhibitory postsynapses within both somata of the granule neurons and its dendrites.

Because it is known that Dp427 is expressed at the inhibitory postsynapses within CA1 pyramidal neurons, we examined whether HADp71 is also present at the inhibitory postsynapses of the CA1 area and revealed that gephyrin-positive synaptic puncta were negative for HA-Dp71 signal while HA-Dp71 was strongly detected at a perivascular glia limitans (Fig. 4a). Both bDg and Dp427 were clearly detected as a synaptic pattern in the CA1 pyramidal cell layer of the TG sections but HA-Dp71 was absent from the synaptic puncta (Fig. 4b, c). Consistent with these results, anti-dystrophin antibody which detects Pan-dystrophin products labelled not only perivascular glia limitans but also synaptic puncta in the CA1 pyramidal neurons, however, anti-HA antibody labelled only glia limitans on the same section (Fig. 4d), indicating that Dp427 and Dp71 are present at different positions, synaptic puncta and glia limitans in the CA1 area, respectively. In the CA1 pyramidal cell layer, Pan-dystrophin-positive synaptic puncta were co-labelled with gephyrin but not with PSD95 (Suppl. Fig. 4), suggesting that Dp427-Dg complex functions at the inhibitory postsynapses within CA1 pyramidal neurons.

\section{HA-Dp71 expression in the inhibitory postsynapse of the cultured hippocampal neurons}

To confirm the existence of Dp71 at inhibitory postsynapse and glial process, we prepared primary hippocampal neuronal culture and primary hippocampal glia culture from TG mouse embryonic hippocampi. Western blots showed that expression levels of HA-Dp71 and bDg were prominent in the cultured glial cells compared to those in the cultured neurons (Fig. 5a). Expressions of HA-Dp71 appeared to be slightly increased upon the neuronal maturation when compared the levels at 10 and 18 days in vitro (DIV) with that at 3 DIV, suggesting that neurite extension and/or synapse formation may enhance Dp71 expression or Dp71 protein stability. PSD95, a mature postsynapse marker, was specifically detected in neurons at later stages, 10 and 18 DIV, whereas TuJ1 (also known as neuron-specific class III b-tubulin) was detected in neurons throughout the cultured period indicated.

We performed double-immunocytochemical stainings of HA-Dp71 with MAP2, Gfap, and bDg in the cultured mature neurons or glial cells (Fig. 5b). HA-Dp71 signals were detected as synaptic puncta within MAP2-positive neuronal soma and its dendrites, whereas gfappositive glial cells had HA-Dp71 signals throughout processes including their edge. bDg was colocalized with HA-Dp71 in both neuronal synaptic puncta and glial processes. We next determined synaptic compartment positive for HA-Dp71 by immunocytochemistry using synapse markers, gephyrin, VGAT, PSD95, and VGLUT1 (Fig. 5c and Suppl. Fig. 5). Remarkably, HA-Dp71 was co-labeled with gephyrin as well as bDg, while VGAT, inhibitory presynapse marker, was adjacent to Dp71-positive puncta. In contrast, excitatory synapse markers, 
PSD95 and VGLUT1, showed no co-labelling with HA-Dp71. We counted the numbers of Dp71-positive synaptic puncta and Gephyrinpositive synaptic puncta and then analyzed Dp71-positive rate on the Gephyrin-positive synapses and Gephyrin-positive rate on the Dp71-positive puncta (Fig. 5d). Among the Gephyrin-positive inhibitory postsynapses, about $43.1 \%$ of synapses had Dp71 expression. When we focused on the Dp71-positive puncta, about 95.9\% were Gephyrin-positive synapses. Next, we counted the numbers of Dp71positive synaptic puncta and PSD95-positive synaptic puncta and then analyzed Dp71-positive rate on the PSD95-positive synapses and PSD95-positive rate on the Dp71-positive puncta (Fig. 5e). Among the PSD95-positive excitatory postsynapses, about $2.5 \%$ of synapses had Dp71 signal. When we focused on the Dp71-positive puncta, about 1.6\% were PSD95-positive synapses. Taken together, HA-Dp71 was exclusively localized to inhibitory postsynapses in the cultured hippocampal neurons. However, HA-Dp71 expression was detected partially but not all the inhibitory postsynapses in the cultured neurons. This result suggested that particular neuronal cell type(s) would express Dp71 and/or particular inhibitory presynaptic innervation(s) would be required for the Dp71-accumulation/stabilization at inhibitory postsynaptic compartment. In agreement with all above results, HA-Dp71 puncta were adjacent to GABAergic presynaptic terminals labelled by GAD67 (Suppl. Fig. 6a). As far as we examined, neither HA-Dp71 detectable neurons or undetectable neurons expressed Dp427 in the cultured hippocampal neurons (Suppl. Fig. 6a), suggesting that Dp71 was the major dystrophin product in the cultured hippocampal neurons.

\section{Dtnb and Dtna are partners for synapse-associated Dp71 and glia limitans-associated Dp71, respectively}

Proteomics analysis is a powerful approach for dissecting protein-protein networks. To explore potential Dp71-interaction proteins especially function at the inhibitory postsynapse in the adult hippocampus, we immunoprecipitated HA-Dp71 molecular complexes from the adult TG mouse hippocampi and identified its components by mass-spectrometry. We found 6 candidates (Dtna, Dtnb, Snta1, Sntb1, Sntb2, and Insyn1) specifically detected in the TG sample by comparing the TG-data with WT-negative control data using a criteria (enrichment $\geq 10$; weighted spectra $>10$ ) (Table 1 and Suppl. Table S2). Among the candidates, dystrobrevins (Dtna and Dtnb) and syntrophins (Snta1, Sntb1 and Sntb2) are well-known as binding partners for Dp427 in muscles and/or Dp71 in brains $[6,9,13]$. Additionally, Insyn 1 was recently reported as a dystrophin-interaction protein at inhibitory postsynapses [34, 35]. As Dtnb was detected at a lower level than the other candidates, especially Dtna, in a similar manner to Insyn1, we speculated that not only Insyn1 but also Dtnb may interact with Dp71 at inhibitory postsynapse of hippocampal dentate gyrus. To address this question, we immunoprecipitated HADp71 by anti-HA magnetic beads from the TG hippocampi and co-precipitated both Dtna and Dtnb (Fig. 6a). Unfortunately, we could not assess the relation between Dp71 and Insyn1 because commercially available anti-Insyn1 antibody did not work at all. As for the relation between Dp71 and bDg, we previously reported that co-immunoprecipitation after cross-linking rather than native coimmunoprecipitation enabled us to detect physical molecular interaction [32]. Indeed, co-immunoprecipitation assay using cross-linked proteins extracted from cultured hippocampal neurons showed physical interaction between Dp71 and bDg (Suppl. Fig. 7). These results indicated that all the three proteins, Dtna, Dtnb and bDg, physically interacted with Dp71 in neurons and/or glia.

Table 1

Potential Dp71-interacting proteins identified by comprehensive mass spectrometry analysis

\begin{tabular}{|lllllll|}
\hline & & & & & Weighted & Spectra \\
\hline Identified Proteins & Alternate ID & Accession \# & Mr (KDa) & Enrichment & TG & WT \\
\hline Dystrophin & Dmd & P11531 & 426 & 145 & 290 & 2 \\
\hline a-1-syntrophin & Snta1 & Q61234 & 54 & INF & 140 & 0 \\
\hline b-1-syntrophin & Sntb1 & Q99L88 & 58 & INF & 112 & 0 \\
\hline Dystrobrevin a & Dtna & Q9D2N4 & 84 & INF & 100 & 0 \\
\hline b-2-syntrophin & Sntb2 & Q61235 & 56 & INF & 38 & 0 \\
\hline Dystrobrevin b & Dtnb & O70585 & 74 & INF & 15 & 0 \\
\hline Inhibitory synaptic factor 1 & Insyn1 & Q8CD60 & 32 & INF & 13 & 0 \\
\hline $\begin{array}{l}\text { Proteins were considered as interaction partners if 1) the weighted spectrum was } \geq 10 \text { and 2) the enrichment value was } \geq 10, \text { where } \\
\text { enrichment was calculated as weighted spectrum (TG)/weighted spectrum (WT). }\end{array}$ \\
\hline
\end{tabular}

We next performed double-immunohistochemistry and indicated that Dtnb and Dtna were co-labelled with HA-Dp71 at synaptic puncta (Fig. 6b) and at glia limitans surrounding BBB in the dentate gyrus (Fig. 6c), respectively. Additionally, Dtnb signals were co-labelled with gephyrin in the dentate gyrus (Fig. 6d). We next examined the expressions of dystrobrevins in the primary hippocampal culture and 
found that Dtnb and Dtna were co-localized with HA-Dp71 at synaptic puncta (Fig. 6e) and at astrocytic processes (Fig. 6f), respectively. Dtnb was co-labelled with gephyrin (Fig. 6g) but not with PSD95 (Fig. 6h), indicating that Dtnb was exclusively present at inhibitory postsynapses. Taken together, Dp71 functions by making complex with Dtnb at the inhibitory postsynapse of dentate gyrus granule neurons and with Dtna at glia limitans.

\section{Discussion}

We generated dystrophin short product (Dp71)-specific tag-insertion mouse line and investigated the expression and localization of endogenous Dp71 protein in the adult brain, especially hippocampus. We detected Dp71, including both Dp71d and Dp71f isoforms, as only dystrophin product derived from intron 62 gene promoter in the adult mouse brains while Dp40 was not detectable at protein level. Dp71 was evidently detected not only in glia limitans but also in the inhibitory postsynapses of hippocampal dentate gyrus granule neurons in vivo, whereas Dp427 was present in the CA1 pyramidal neurons. We unveiled distinct Dp71 glycoprotein complexes, i.e. synapse-associated Dp71 interacted with Dtnb and Dg, in contrast, glia-associated Dp71 did with Dtna and Dg (Fig. 7).

Dystrophin gene produces multiple products, Dp427, Dp260, Dp140, Dp116 and Dp71, from different promoters in tissue-type dependent manner [6, 9]. Dp71 is the major product from intron 62 promoter, and alternative splicing potentially provides different Dp71 isoforms including Dp40 shortest isoform $[9,28]$. Although mRNA analyses to clarify the expression profiles of $d y$ strophin in tissues and cell lines had been performed $[17,19,28,29]$, a convincing answer is missing. One difficulty is that anti-Dp71 antibodies also recognize longer dystrophin products and its degraded products. Indeed, we privately observed that Dp71 was prominently digested by calciumdependent protease, calpain, and degraded into shorter products. When we previously prepared brain extracts without calcium chelator such as EDTA, particular digested product derived from Dp71 was detected around $40 \mathrm{kDa}$ on western blots [33]. Here, we utilized the tag-insertion mice in combination with specific HA-antibody and revealed that Dp71 but not Dp40 was detectable at protein levels in the adult brains. The fact that Dp71 was also detected in the muscle extracts from TG mice indicated that detection efficiency against HADp71 was excellent. Since we and others had detected Dp40 mRNA expression in mouse brains $[33,36]$, its translational efficacy must be extremely low and under the limit of detection by standard techniques. However, we do not deny the possibility that Dp40 would be expressed in particular cell-types and/or conditions. Additionally, it is impossible to distinguish expressions of different Dp71 isoforms immunohistochemically by tag-detection in the transgenic mouse tissues because the HA-tag was inserted at the N-terminus of Dp71 sequence. Thus, we herein characterized spatial expressions of Pan-Dp71 in the adult mouse brains and primary cultured cells.

It is a remarkable observation that Dp71 was detected within the hippocampal dentate gyrus and was specialized in the inhibitory postsynapse compartment of granule neurons. Since inhibitory postsynaptic localization of Dp71 was detectable in both dentate gyrus granular layer and molecular layer, Dp71 would be relevant to the perisomatic and dendritic postsynaptic functions. Interestingly, Dp71 was not detectable in the CA1 pyramidal layer, where Dp427 was prominently present, indicating that dystrophin gene expressions were regulated in cell-type dependent manner. As there are reports suggesting preferential expressions of Dp71 mRNA in the dentate gyrus granule neurons and of Dp427 mRNA in the CA pyramidal neurons $[15,16]$, we interpret that our data provides direct evidence showing spatial Dp71 and Dp427 protein expressions in the hippocampus. Specific transcription factors possibly drive different gene promoters within the dystrophin gene to produce proper products in each cell-type. Therefore, promoter analysis based on transcription factor binding sites in combination with gene expression analysis may help us to understand the comprehensive regulatory mechanism of dystrophin expression in the future.

We addressed subcellular localization of Dp71 among synapse compartments, excitatory-/inhibitory- and pre-/post-synapses, in the primary cultured neurons and found exclusive localization of Dp71 at the inhibitory postsynapses. Dp71 was not detected in the other subcellular compartments such as nucleus. Although western blot analysis demonstrated Dp71 expression in both immature and mature neuronal cultures, Dp71 became detectable by immunocytochemistry only in the mature neurons, especially at the synapses. Dp71-positive puncta were co-labelled with gephyrin but not with PSD95, and GAD67-positive and VGAT-positive GABAergic presynapses were adjacent to $\mathrm{Dp} 71$ puncta. Colocalization of bDg with $\mathrm{Dp} 71$ in the cultured neurons as well as in dentate granule neurons indicated that Dp71 functions together with bDg at inhibitory postsynapses. Our data is well-consistent with the previous report that Dg was selectively associated with inhibitory postsynapses in the hippocampal neurons and conditional-knockout of Dg in the neurons revealed reduction of dystrophin which was detected by anti-dystrophin C-terminal antibody [37]. Since we previously showed that Dg is required for the proper expression and submembranous localization of Dp71 in cultured cells and physical protein-protein interaction mediates this process [32], Dg possibly anchors Dp71 at inhibitory postsynapses and provides a molecular scaffold to form a functional complex. We also considered the following possibilities: Dp71 was diffusely distributed in the dendrites and soma at low levels during immature stage, and was accumulated and stabilized at the inhibitory postsynapse compartment. Alternatively, as neuronal culture included glial 
cells at low populations, glia-derived Dp71 may reveal overestimation of Dp71 expression levels in the immature neuronal extracts by western blot analysis. As far as we examined, Dp71 was detected at not all, but part of the gephyrin-positive synapses, suggesting that proper signal is necessary for Dp71 clustering at specialized compartment, or particular cell-types express Dp71 in the heterogenous neuronal culture. Since the hippocampal neuronal culture includes at least pyramidal neurons, granule neurons, interneurons and glial cells [38], proper presynaptic innervations from particular GABAergic neurons might reflect physiological situation. Because Dp427 was not detected by Dys1 antibody in our culture system, Dp71 expression must be dominant in vitro, which is possibly regulated by promoter usage. As it remains elusive which cell-types express Dp71 in the heterogenous neuronal culture, it is valuable to further assess the exact regulatory mechanism of dystrophin expressions at specialized synapses in the future. From this view point, it is intriguing that conditional deletion of Dg in pyramidal neurons caused loss of CCK-positive basket cell terminals in hippocampus and neocortex [39], which let us speculate that Dp427-Dg complex and Dp71-Dg complex would be necessary for formation and maintenance of CCKpositive terminals on pyramidal neurons and dentate gyrus granule neurons in hippocampus, respectively.

The Dp71-specific tag-insertion mouse line is a useful experimental tool to clarify the molecular basis of Dp71 complex in vivo since biochemical analyses revealed dystrobrevins, syntrophins and Insyn1 as binding partners of Dp71 in the adult mouse hippocampus. Due to the limitations of specific materials for Insyn1 and syntrophins, we herein focused on the molecular analyses of dystrobrevins and found that Dtna and Dtnb are associated with Dp71 at glia foot process and inhibitory postsynapse, respectively. Blake DJ et al. indicated that Dp427 interacts with Dtnb at neuronal synapses whereas Dp71 does with Dtna in glia and further gave thoughtful consideration that Dp71 may function with Dtnb in the dentate gyrus granule neurons [24]. Our data provided the direct evidence to support their idea and reminded us the previous report that cerebellar Purkinje cells and Bergmann glia cells expressed Dtnb and Dtna, respectively [40]. However, relationship among Dp71 and dystrobrevins in the cerebellum and cerebral cortex should await future study.

Recently, Insyn1 (also known as C15orf59) was identified and characterized as an inhibitory synapse-specialized proteins, which directly associated with dystrophin-Dg complex [34, 35]. Insyn1 is a potential candidate gene for epilepsy and intellectual deficits [41]. In consistent with our data, Uezu A et al. showed that Insyn1 coprecipitated dystrophin, Dtna, Dtnb, Snta1, Sntb1 and Sntb2 [34]. Given the fact, it is highly possible that Dp71 functions together with Dtnb, Insyn1, and syntrophins as synapse-associated Dp71 glycoprotein complex in the hippocampal granule neurons (Fig. 7). As for the glia-associated Dp71 glycoprotein complex, Dp71 was reported to be involved in aquaporin-4 (AQP4) water channel system by indirect interaction via Snta1 [25-27, 42]. Thus, Dp71, Dtna and Snta1 would bridge two different integral proteins, Dg and AQP4.

Dp71-specific functions have been investigated by using Dp71-deficient mice [23, 43], demonstrating that Dp71 is involved in a broad range of synaptic regulations in the hippocampal CA1 field possibly thorough alterations of both pre- and post-synaptic mechanisms. Daoud $\mathrm{F}$ et al. suggested that Dp71f isoform was expressed at the excitatory postsynapses in hippocampal neuronal culture and biochemically fractionated in the post-synaptic density fraction [23]. Rodríguez-Muñoz R et al. reported Dp71 localization at nucleoskeleton in the cultured neurons [44]. In contrast, we herein detected exclusive expression of Dp71 at inhibitory postsynapses, although HA-tag detection provided both Dp71d and Dp71f signals. Similarly, Pan-dystrophin antibody showed inhibitory postsynaptic distribution in the CA1 pyramidal layer. Thus, although there might be differences in cell culture condition or detection efficiency among the different studies, it remains possible that anti-Dp71 antibodies cross-react against dystrophin-related or non-related proteins. Additionally, in accordance with the previous notion that general biochemical fractionations are incomplete and post-synaptic density fractions include glia-derived proteins [24], we privately observed a significant amount of contamination of glial fibrillary acidic protein in the synaptosome fraction. Therefore, further evaluation on both present and previous results are necessary to precisely understand Dp71 expressions and functions.

As a dystrophin reporter mouse line, Dmd-FLAG-EGFP was previously established by Petkova MV et al. and used to investigate dystrophin(s) expression [45]. In this line, FLAG-EGFP tandem tag was fused with the C-terminus of dystrophin(s) located within the last exon 79 so that Pan-dystrophin was detectable. Dmd-FLAG-EGFP signal was detected at glia limitans, which corresponded to Dp71 expression, and at hippocampal CA1 pyramidal and cerebellar Purkinje cells, which corresponded to Dp427, however, there was no description of dystrophin expression in the hippocampal granule neurons [45]. Comparative experiments using two distinct mouse lines, Dmd-FLAG-EGFP and our HA-Dp71, would reveal better understandings of dystrophin(s) expressions.

In this study, we established Dp71-specific tag-insertion mice to visualize endogenous Dp71 expression and localization in the adult mouse hippocampus and demonstrated that Dp71 was expressed abundantly at glia limitans and exclusively at inhibitory postsynapse of hippocampal granule neurons. Synapse-associated Dp71 glycoprotein complex had a tight relation with Dtnb and Insyn1. In the 
future, we will extend our work to several fields, investigations of Dp71 profiles in the other brain regions, cerebral cortex and cerebellum, and in developing brains and peripheral tissues.

\section{Declarations}

Acknowledgements We thank the past and present members of K.I.'s laboratory for fruitful discussions.

Author contributions Conceptualization: TF, Kl; Methodology: TF; Formal analysis and investigation: TF, TY; Writing - original draft preparation: TF; Writing - review and editing: TF, Kl; Funding acquisition: TF, TO; Resources: KN, TA, TO, Kl; Supervision: KI. All authors read and approved the final manuscript.

Funding This work was supported by grants from MEXT KAKENHI (18K07883 and 21K07279) to TF, and partially by Grants-in-Aid for Research from the National Center for Global Health and Medicine (20A1019 and 21A1018) to TO.

Data Availability The datasets generated during and/or analyzed during the current study are available from the corresponding author on reasonable request. Supporting data can also be found in the Supplement to this manuscript.

Conflict of interest The authors state no conflicts of interest.

Ethics approval All animal experiments were approved by the President of Kyoto Prefectural University and the National Center for Global Health and Medicine following consideration by the Institutional Animal Care and Use Committee of each institute (approval ID: M30519, M2020-241-1, M2021-268 and 18037), and were carried out in accordance with institutional procedures, national guidelines, and the relevant national laws on the protection of animals.

\section{References}

1. Deburgrave N, Daoud F, Llense S, Barbot JC, Récan D, Peccate C, Burghes AH, Béroud C, Garcia L, Kaplan JC, Chelly J, Leturcq F (2007) Protein- and mRNA-based phenotype-genotype correlations in DMD/BMD with point mutations and molecular basis for BMD with nonsense and frameshift mutations in the DMD gene. Hum Mutat 28(2):183-195. https://doi.org/10.1002/humu.20422

2. Koenig M, Hoffman EP, Bertelson CJ, Monaco AP, Feener C, Kunkel LM (1987) Complete cloning of the Duchenne muscular dystrophy (DMD) CDNA and preliminary genomic organization of the DMD gene in normal and affected individuals. Cell 50(3):509517. https://doi.org/10.1016/0092-8674(87)90504-6

3. Kunkel LM, Hejtmancik JF, Caskey CT et al (1986) Analysis of deletions in DNA from patients with Becker and Duchenne muscular dystrophy. Nature 322(6074):73-77. https:// doi.org/10.1038/322073a0

4. Juan-Mateu J, Gonzalez-Quereda L, Rodriguez MJ, Baena M, Verdura E, Nascimento A, Ortez C, Baiget M, Gallano P (2015) DMD Mutations in 576 Dystrophinopathy Families: A Step Forward in Genotype-Phenotype Correlations. PLoS One 10(8):e0135189. https://doi.org/10.1371/journal.pone.0135189

5. Tuffery-Giraud S, Béroud C, Leturcq F et al (2009) Genotype-phenotype analysis in 2,405 patients with a dystrophinopathy using the UMD-DMD database: a model of nationwide knowledgebase. Hum Mutat 30(6):934-945.https://doi.org/10.1002/humu.20976

6. Blake DJ, Kröger S (2000) The neurobiology of duchenne muscular dystrophy: learning lessons from muscle? Trends Neurosci 23(3):92-99. https://doi.org/10.1016/S0166-2236(99)01510-6

7. Dalloz C, Sarig R, Fort P, Yaffe D, Bordais A, Pannicke T, Grosche J, Mornet D, Reichenbach A, Sahel J, Nudel U, Rendon A (2003) Targeted inactivation of dystrophin gene product Dp71: phenotypic impact in mouse retina. Hum Mol Genet 12(13):1543-1554. https://doi.org/10.1093/hmg/ddg170

8. Hendriksen RGF, Vles JSH, Aalbers MW, Chin RFM, Hendriksen JGM (2018) Brain-related comorbidities in boys and men with Duchenne Muscular Dystrophy: A descriptive study. Eur J Paediatr Neurol 22(3):488-497. https://doi.org/10.1016/j.ejpn.2017.12.004

9. Naidoo M, Anthony K (2020) Dystrophin Dp71 and the Neuropathophysiology of Duchenne Muscular Dystrophy. Mol Neurobiol 57(3):1748-1767. https://doi.org/10.1007/s12035-019-01845-w

10. Pane M, Messina S, Bruno C, D'Amico A, Villanova M, Brancalion B, Sivo S, Bianco F, Striano P, Battaglia D, Lettori D, Vita GL, Bertini E, Gualandi F, Ricotti V, Ferlini A, Mercuri E (2013) Duchenne muscular dystrophy and epilepsy. Neuromuscul Disord 23(4):313-315. https://doi.org/10.1016/j.nmd.2013.01.011 
11. Sene A, Tadayoni R, Pannicke T, Wurm A, El Mathari B, Benard R, Roux MJ, Yaffe D, Mornet D, Reichenbach A, Sahel JA, Rendon A (2009) Functional implication of Dp71 in osmoregulation and vascular permeability of the retina. PLoS One 4(10):e7329. https://doi.org/10.1371/journal.pone.0007329

12. Waite A, Brown SC, Blake DJ (2012) The dystrophin-glycoprotein complex in brain development and disease. Trends Neurosci 35(8):487-496. https://doi.org/10.1016/j.tins.2012.04.004

13. Peters MF, Adams ME, Froehner SC (1997) Differential association of syntrophin pairs with the dystrophin complex. J Cell Biol 138(1):81-93. https://doi.org/10.1083/jcb.138.1.81

14. Doorenweerd N, Mahfouz A, van Putten M, Kaliyaperumal R, T' Hoen PAC, Hendriksen JGM, Aartsma-Rus AM, Verschuuren JJGM, Niks EH, Reinders MJT, Kan HE, Lelieveldt BPF (2017) Timing and localization of human dystrophin isoform expression provide insights into the cognitive phenotype of Duchenne muscular dystrophy. Sci Rep 7(1):12575. https://doi.org/10.1038/s41598-01712981-5

15. Górecki DC, Abdulrazzak H, Lukasiuk K, Barnard EA (1997) Differential expression of syntrophins and analysis of alternatively spliced dystrophin transcripts in the mouse brain. Eur J Neurosci 9(5):965-976. https://doi.org/10.1111/j.1460-9568.1997.tb01447.x

16. Górecki DC, Barnard EA (1995) Specific expression of G-dystrophin (Dp71) in the brain. Neuroreport 6(6):893-896. https://doi.org/10.1097/00001756-199504190-00017

17. Hugnot JP, Gilgenkrantz H, Vincent N, Chafey P, Morris GE, Monaco AP, Berwald-Netter Y, Koulakoff A, Kaplan JC, Kahn A, Chelly J (1992) Distal transcript of the dystrophin gene initiated from an alternative first exon and encoding a 75-kDa protein widely distributed in nonmuscle tissues. Proc Natl Acad Sci U S A 89(16):7506-7510. https://doi.org/10.1073/pnas.89.16.7506

18. Knuesel I, Mastrocola M, Zuellig RA, Bornhauser B, Schaub MC, Fritschy JM (1999) Short communication: altered synaptic clustering of GABAA receptors in mice lacking dystrophin (mdx mice). Eur J Neurosci 11(12):4457-4462. https://doi.org/10.1046/j.1460-9568.1999.00887.x

19. Lederfein D, Levy Z, Augier N, Mornet D, Morris G, Fuchs O, Yaffe D, Nudel U (1992) A 71-kilodalton protein is a major product of the Duchenne muscular dystrophy gene in brain and other nonmuscle tissues. Proc Natl Acad Sci U S A 89(12):5346-5350. https://doi.org/10.1073/pnas.89.12.5346

20. Lidov HG, Byers TJ, Watkins SC, Kunkel LM (1990) Localization of dystrophin to postsynaptic regions of central nervous system cortical neurons. Nature 348(6303):725-728. https://doi.org/10.1038/348725a0

21. Moukhles H, Carbonetto S (2001) Dystroglycan contributes to the formation of multiple dystrophin-like complexes in brain. $J$ Neurochem 78(4):824-834. https://doi.org/10.1046/j.1471-4159.2001.00466.x

22. Uchino M, Yoshioka K, Miike T, Tokunaga M, Uyama E, Teramoto H, Naoe H, Ando M (1994) Dystrophin and dystrophinrelated protein in the brains of normal and mdx mice. Muscle Nerve 17(5):533-538. https://doi.org/10.1002/mus.880170511

23. Daoud F, Candelario-Martínez A, Billard JM, Avital A, Khelfaoui M, Rozenvald Y, Guegan M, Mornet D, Jaillard D, Nudel U, Chelly J, Martínez-Rojas D, Laroche S, Yaffe D, Vaillend C (2008) Role of mental retardation-associated dystrophin-gene product Dp71 in excitatory synapse organization, synaptic plasticity and behavioral functions. PLoS One 4(8):e6574. https://doi.org/10.1371/journal.pone.0006574

24. Blake DJ, Hawkes R, Benson MA, Beesley PW (1999) Different dystrophin-like complexes are expressed in neurons and glia. J Cell Biol 147(3):645-658. https://doi.org/10.1083/jcb.147.3.645

25. Belmaati Cherkaoui M, Vacca O, Izabelle C, Boulay AC, Boulogne C, Gillet C, Barnier JV, Rendon A, Cohen-Salmon M, Vaillend C (2021) Dp71 contribution to the molecular scaffold anchoring aquaporine-4 channels in brain macroglial cells. Glia 69(4):954-970. https://doi.org/10.1002/glia.23941

26. Haenggi T, Fritschy JM (2006) Role of dystrophin and utrophin for assembly and function of the dystrophin glycoprotein complex in non-muscle tissue. Cell Mol Life Sci 63(14):1614-1631. https://doi.org/10.1007/s00018-005-5461-0

27. Haenggi T, Soontornmalai A, Schaub MC, Fritschy JM (2004) The role of utrophin and Dp71 for assembly of different dystrophinassociated protein complexes (DPCs) in the choroid plexus and microvasculature of the brain. Neuroscience 129(2):403-413. https://doi.org/10.1016/j.neuroscience.2004.06.079

28. Aragón J, González-Reyes M, Romo-Yáñez J, Vacca O, Aguilar-González G, Rendón A, Vaillend C, Montañez C (2018) Dystrophin Dp71 Isoforms Are Differentially Expressed in the Mouse Brain and Retina: Report of New Alternative Splicing and a Novel Nomenclature for Dp71 Isoforms. Mol Neurobiol 55(2):1376-1386. https://doi.org/10.1007/s12035-017-0405-x 
29. Schofield JN, Blake DJ, Simmons C, Morris GE, Tinsley JM, Davies KE, Edwards YH (1994) Apo-dystrophin-1 and apo-dystrophin-2, products of the Duchenne muscular dystrophy locus: expression during mouse embryogenesis and in cultured cell lines. Hum Mol Genet 3(8):1309-1316. https://doi.org/10.1093/hmg/3.8.1309

30. Aida T, Chiyo K, Usami T, Ishikubo H, Imahashi R, Wada Y, Tanaka KF, Sakuma T, Yamamoto T, Tanaka K (2015) Cloning-free CRISPR/Cas system facilitates functional cassette knock-in in mice. Genome Biol 16(1):8. https://doi.org/10.1186/s13059-0150653-x

31. Kawai S, Takagi Y, Kaneko S, Kurosawa T (2011) Effect of three types of mixed anesthetic agents alternate to ketamine in mice. Exp Anim 60: 481-487. https://doi.org/10.1538/expanim.60.481

32. Fujimoto T, Yaoi T, Tanaka H, Itoh K (2020) Dystroglycan regulates proper expression, submembranous localization and subsequent phosphorylation of Dp71 through physical interaction. Hum Mol Genet 29(19):3312-3326. https://doi.org/10.1093/hmg/ddaa217

33. Fujimoto T, Itoh K, Yaoi T, Fushiki S (2014) Somatodendritic and excitatory postsynaptic distribution of neuron-type dystrophin isoform, Dp40, in hippocampal neurons. Biochem Biophys Res Commun 452(1):79-84. https://doi.org/10.1016/j.bbrc.2014.08.064

34. Uezu A, Kanak DJ, Bradshaw TW, Soderblom EJ, Catavero CM, Burette AC, Weinberg RJ, Soderling SH (2016) Identification of an elaborate complex mediating postsynaptic inhibition. Science 353(6304):1123-1129. http://doi.org/10.1126/science.aag0821

35. Uezu A, Hisey E, Kobayashi Y, Gao Y, Bradshaw TW, Devlin P, Rodriguiz R, Tata PR, Soderling S (2019) Essential role for InSyn1 in dystroglycan complex integrity and cognitive behaviors in mice. Elife 8:e50712. https://doi.org/10.7554/eLife.50712

36. Paúl-González S, Aragón J, Rodríguez-Martínez G, Romo-Yáñez J, Montanez C (2021) Differential expression of Dp71 and Dp40 isoforms in proliferating and differentiated neural stem cells: Identification of Dp40 splicing variants. Biochem Biophys Res Commun 560:152-158. https://doi.org/10.1016/j.bbrc.2021.03.142

37. Lévi S, Grady RM, Henry MD, Campbell KP, Sanes JR, Craig AM (2002) Dystroglycan is selectively associated with inhibitory GABAergic synapses but is dispensable for their differentiation. J Neurosci 22(11):4274-4285. https://doi.org/10.1523/JNEUROSCI.22-11-04274.2002

38. Wu YK, Fujishima K, Kengaku M (2015) Differentiation of apical and basal dendrites in pyramidal cells and granule cells in dissociated hippocampal cultures. PLoS One 10(2):e0118482. https://doi.org/10.1371/journal.pone.0118482

39. Früh S, Romanos J, Panzanelli P, Bürgisser D, Tyagarajan SK, Campbell KP, Santello M, Fritschy JM (2016) Neuronal Dystroglycan Is Necessary for Formation and Maintenance of Functional CCK-Positive Basket Cell Terminals on Pyramidal Cells. J Neurosci 36(40):10296-10313. https://doi.org/10.1523/JNEUROSCI.1823-16.2016

40. Grady RM, Wozniak DF, Ohlemiller KK, Sanes JR (2006) Cerebellar synaptic defects and abnormal motor behavior in mice lacking alpha- and beta-dystrobrevin. J Neurosci 26(11):2841-2851. https://doi.org/10.1523/JNEUROSCI.4823-05.2006

41. Huynh MT, Lambert AS, Tosca L, Petit F, Philippe C, Parisot F, Benoît V, Linglart A, Brisset S, Tran CT, Tachdjian G, Receveur A (2018) 15q24.1 BP4-BP1 microdeletion unmasking paternally inherited functional polymorphisms combined with distal 15q24.2q24.3 duplication in a patient with epilepsy, psychomotor delay, overweight, ventricular arrhythmia. Eur J Med Genet 61(8):459-464. https://doi.org/10.1016/j.ejmg.2018.03.005

42. Neely JD, Amiry-Moghaddam M, Ottersen OP, Froehner SC, Agre P, Adams ME (2001) Syntrophin-dependent expression and localization of Aquaporin-4 water channel protein. Proc Natl Acad Sci U S A 98(24):14108-14113. https://doi.org/10.1073/pnas.241508198

43. Miranda R, Nudel U, Laroche S, Vaillend C (2011) Altered presynaptic ultrastructure in excitatory hippocampal synapses of mice lacking dystrophins Dp427 or Dp71. Neurobiol Dis 43(1):134-141. https://doi.org/10.1016/j.nbd.2011.02.017

44. Rodríguez-Muñoz R, Cárdenas-Aguayo Mdel C, Alemán V, Osorio B, Chávez-González O, Rendon A, Martínez-Rojas D, Meraz-Ríos MA (2015) Novel Nuclear Protein Complexes of Dystrophin 71 Isoforms in Rat Cultured Hippocampal GABAergic and Glutamatergic Neurons. PLoS One 10(9):e0137328. https://doi.org/10.1371/journal.pone.0137328

45. Petkova MV, Morales-Gonzales S, Relizani K, Gill E, Seifert F, Radke J, Stenzel W, Garcia L, Amthor H, Schuelke M (2016) Characterization of a Dmd (EGFP) reporter mouse as a tool to investigate dystrophin expression. Skelet Muscle 6:25. https://doi.org/10.1186/s13395-016-0095-5

\section{Supplementary Files}

This is a list of supplementary files associated with this preprint. Click to download. 
- Suppl.Fig.pdf

- Suppl.TableS2.xlsx

Page $12 / 12$ 